\title{
BMJ Open Willingness, perceived barriers and motivators in adopting mobile applications for health-related interventions among older adults: a scoping review protocol
}

\author{
Nurul Asilah Ahmad (D , ${ }^{1}$ Arimi Fitri Mat Ludin, ${ }^{1}$ Suzana Shahar, \\ Shahrul Azman Mohd Noah, ${ }^{2}$ Noorlaili Mohd Tohit $^{3}$
}

To cite: Ahmad NA, Mat Ludin AF, Shahar S, et al. Willingness, perceived barriers and motivators in adopting mobile applications for health-related interventions among older adults: a scoping review protocol. BMJ Open 2020;10:e033870. doi:10.1136/ bmjopen-2019-033870

- Prepublication history and additional material for this paper are available online. To view these files, please visit the journal online (http://dx.doi. org/10.1136/bmjopen-2019033870).

Received 25 September 2019 Revised 06 February 2020 Accepted 19 February 2020
Check for updates

(C) Author(s) (or their employer(s)) 2020. Re-use permitted under CC BY-NC. No commercial re-use. See rights and permissions. Published by BMJ.

For numbered affiliations see end of article.

Correspondence to Dr Arimi Fitri Mat Ludin; arimifitri@ukm.edu.my

\section{ABSTRACT}

Introduction The world's older population continues to grow at an unprecedented rate. An ageing population poses a great challenge to our healthcare system that requires new tool to tackle the complexity of health services as well as the increasing expenses. Mobile health applications (mHealth app) is seen to have the potential to address these challenges, alleviating burdens on the healthcare system and enhance the quality of life for older adults. Despite the numerous benefits of mHealth apps, relatively little is known about whether older adults perceive that these apps confer such benefits. Their perspectives towards the use of mobile applications for health-related purposes have also been little studied. Therefore, in this paper, we outline our scoping review protocol to systematically review literature specific to older adults' willingness, perceived barriers and motivators towards the use of mobile applications to monitor and manage their health.

Methods and analysis Arksey and 0'Malley's scoping review methodology framework will guide the conduct of this scoping review. The search strategy will involve electronic databases including PubMed, Excerpta Medica Database, Cumulative Index of Nursing and Allied Health Literature, Cochrane Library, Google Scholar and ScienceDirect, in addition to grey literature sources and hand-searching of reference lists. Two reviewers will independently screen all abstracts and full-text studies for inclusion. Data will be charted and sorted through an iterative process by the research team. The extracted data will undergo a descriptive analysis and simple quantitative analysis will be conducted using descriptive statistics. Engagement with relevant stakeholders will be carried out to gain more insights into our data from different perspectives.

Ethics and dissemination Since the data used are from publicly available sources, this study does not require ethical approval. Results will be disseminated through academic journals, conferences and seminars. We anticipate that our findings will aid technology developers and health professionals working in the area of ageing and rehabilitation.

\section{Strengths and limitations of this study}

- This scoping review will capture current issues and opportunities related to technology-enabled mobile applications among older adults.

- Preferred Reporting Items for Systematic Reviews and Meta-Analyses extension for Scoping Review tool will be used to ensure a systematic approach to searching, screening, charting, collating, reporting and stakeholders consultation.

- The search strategy is comprehensive and includes both peer-reviewed literature (electronic bibliographic databases) and grey literature.

- Despite the strength, this scoping review only considers studies written in English where large number of studies in other languages will be missed out.

- As this is a scoping review, critical appraisal of the study quality and the risk of bias will not be undertaken.

\section{INTRODUCTION}

The world's older population continues to grow at a rapid pace. Today, there are 703 million people aged 65 years or over in the world. ${ }^{1}$ This number is projected to double to 1.5 billion in 2050 with the proportion of one in six people in the world will be aged 65 years or over. ${ }^{1}$ In the case of Malaysia's population, this subpopulation has increased gradually since the 1970 s and expected to be tripled from 2.0 million today to more than 6.0 million by $2040 .^{2} 3$ This phenomenon represents one of the remarkable achievement of mankind history with respect to health, social and economic improvements over time. ${ }^{1}$ The improvements in healthcare system such as infections control, immunisations and better access in healthcare are among the huge contributors to the sustained increases in life expectancy across the globe. ${ }^{4-6}$ 
However, this success history of human life expectancy did not come with a proportionate increase in quality of life for older adults. As heavily discussed in the literature, increased life expectancy has increased the risk in developing chronic diseases, disability and dementia prior to death. ${ }^{78}$ This explains a higher use of health services and greater demand for specialised services among the elderly. ${ }^{9-11}$ Consequently, this puts increasing pressure on the economy and social systems in most countries due to the complexity of health services required along with increased health expenditure. ${ }^{12-14}$

Technological innovations have enabled us to carry out tasks effectively and efficiently. The field of technology-supported healthcare is remarkably growing and provide new ways of self-management and support. Although older adults may be seen as technological laggards, the internet usage among this subpopulation has been reported to increase from year to year. ${ }^{15}$ For instance, in the UK, the internet usage among older adults aged 65-74 group has increased gradually over the last 8 years, with $52 \%$ in 2011 to $83 \%$ in $2019 .{ }^{16}$ To add, the trend of smartphone ownership reported to grow rapidly across the globe. ${ }^{17}$

This rapid growth of technology, particularly in smartphones and internet use, has led to a surge of interest in using mobile applications as a tool to seek health information as well as to monitor and manage health (commonly known as mobile health or mHealth) ${ }^{18-20}$ mHealh is defined as 'medical and public health practice supported by mobile devices, personal digital assistants and other wireless devices'. ${ }^{21}$ There are more than 325000 identified mHealth applications covering diverse of health, fitness and medical topics. ${ }^{22}{ }^{23}$ There is clear evidence that mHealth applications is effective in improving self-care, self-management, self-efficacy, medication adherence as well as in improving health behaviours such as quality of sleep, diet, physical activity and mental health. ${ }^{24}$ In particular to older adults population, there are a number of studies demonstrating the benefits of mHealth towards older adults. ${ }^{25-29}$ This includes, it can help to address existing barriers to treatment such as long waiting time at hospital, poor access to transportation and increased cost of healthcare services. ${ }^{25-29}$

The steady growth of older adult population combined with rising trend in technology uptake within this subpopulation suggest mHealth applications may represent a novel way to improve the health of older adults as well as to reduce healthcare cost. Despite the numerous benefits of mHealth applications, ${ }^{30-33}$ relatively little is known about whether older adults perceive that these apps confer such benefits. Their perspectives towards the use of mobile applications for health-related purposes have also been little studied. Therefore, this review aims to identify what is known about the perspectives in adopting mobile applications for health-related interventions among older adults. The specific research questions are:
1. What is the level of willingness among older adults in using mobile applications to monitor and manage their health conditions?

2. What are the existing barriers among older adults in using mobile applications to monitor and manage their health conditions?

3. What motivates older adults to use mobile applications to monitor and manage their health conditions?

\section{METHODS AND ANALYSIS}

\section{Protocol development}

This study will adopt Arksey and O'Malley's ${ }^{34}$ framework for scoping reviews as the foundation and more recent advancements to the methodology ${ }^{35-37}$ as well as the updated framework by the Joanna Briggs Institute. ${ }^{38}$ According to this framework, there are six different stages which includes ${ }^{1}$; identifying the research question, ${ }^{2}$ identifying relevant studies, ${ }^{3}$ selecting studies, ${ }^{4}$ charting the data, ${ }^{5}$ collating, summarising and reporting results and ${ }^{6}$ consulting with stakeholders. The scoping review will also adhere to the Preferred Reporting Items for Systematic Reviews and Meta-Analyses Extension for Scoping Reviews (PRISMA-ScR) ${ }^{39}$ The PRISMA-ScR checklist is attached as online supplementary file 1. International Prospective Register of Systematic Reviews registration is not required as it is a scoping review.

\section{Stage 1: identifying the research question}

Arksey and O'Malley ${ }^{34}$ describe the definition of a relevant research question as a crucial initial step that define and refines the chosen research strategy. We have identified one overarching research question to guide our systematic search strategy and reporting of results: 'What is known about the perspectives in adopting mobile applications for health-related interventions among older adults?'. We aim to provide answers for the following subquestions:

1. What is the level of willingness among older adults in using mobile applications to monitor and manage their health conditions?

2. What are the existing barriers among older adults in using mobile applications to monitor and manage their health conditions?

3. What motivates older adults to use mobile applications to monitor and manage their health conditions?

\section{Stage 2: identifying relevant studies}

The search strategy was collaboratively developed by our research team. To determine the relevance of the citations and to resolve any potential disagreements, the research team will meet to refine the study inclusion and exclusion criteria prior to assessing the articles independently. Our literature search is open, including both peer-reviewed literature as well as grey literature, that is, evidence not published in peer-reviewed publications and from the first 10 pages in the Google search engine.

The identification of relevant literature will consist of three-stage approach. The first stage is searching the 
Table 1 List of keywords and synonyms generated as search terms

\begin{tabular}{|c|c|c|c|c|}
\hline Mobile application & Older adults & Perspective & Barrier & Facilitates \\
\hline Mobile app* & Elderly & View & Limitation & Motivate* \\
\hline mHealth & Ageing population & Attitude & Difficulty & Promote $^{*}$ \\
\hline Telehealth & Ageing & Willingness & Drawback & Ease \\
\hline Mobile technolog* & Geriatric & Readiness & & Aid \\
\hline
\end{tabular}

electronic databases using standardised search terms adapted to the requirements of each respective database. The following electronic databases have been selected ${ }^{1}$ : PubMed $^{2}$; Excerpta Medica Database ${ }^{3}$; Cumulative Index to Nursing and Allied Health Literature ${ }^{4}$; Cochrane Library $^{5}$; Google Scholar and ${ }^{6}$ ScienceDirect. To achieve the level of comprehensiveness required for scoping review, we will also hand search key electronic journals, including the Journal of the American Medical Informatics Association, the Journal of Medical Internet Research, the International Journal of Digital Healthcare, Digital Health and the Journal of mHealth. The second stage involves searching the reference lists of literature that meet all inclusion criteria. The third and final stage involves hand searching specific key publications such as identified white papers or conference presentations for any references we may have missed. We will search relevant grey literature databases (eg, Grey Literature Report, OpenGrey, Web of Science Conference Proceedings, Government Document, academic thesis/dissertation) to identify studies, reports and conference abstracts of relevance to this review.

Search terms from key words, subject heading and synonyms such as mobile application*, mobile app*, mhealth, mobile health, telehealth, mobile technolog*, older adult*, elder*, ageing population, older population, aging, geriatric, perspective, view, attitude, mindset, willingness, readiness, acceptability, barrier, limitation, difficulty, restriction, drawback, facilitate*, motivate*, promote*, help, ease, aid will be generated by the research team members to capture any potential resources from the databases. Table 1 outlines the initial keywords and search terms generated. Boolean operators (AND, OR, NOT) will be used to combine search terms within related keywords. An additional search will be carried out using updated search terms if there are any search terms were missing. table 2 shows the search strings generated.

\section{Stage 3: study selection}

The third stage of Arksey and O'Malley's framework ${ }^{34}$ aims to identify the studies that will be included in the scoping review. The screening process will consist of two stages ${ }^{1}$ : a title and abstract/summary and ${ }^{2}$ full-text screening.

In the first stage, two reviewers will independently screen the titles and abstract of the articles where during this stage, the following decisions will be undertaken ${ }^{1}$ : for any article that both reviewers agree to include, the article will proceed onto the second stage of screening process where the article will be read in full by each reviewer ${ }^{2}$; for any article that both reviewers agree to exclude, the article will not be read in full and excluded from the study $^{3}$; for any article that did not achieved agreement between both reviewers that is, whether to include or exclude, the article will proceed onto the second stage of screening process to be read in full by each reviewer before final decision is made. In the second stage, both reviewers will independently perform a full-text review of the included articles. Disagreements regarding eligibility of sampled articles will be discussed between the two reviewers until consensus is reached or by arbitration of a third reviewer, if required.

Table 2 List of search strings

"Mobile application*" OR "mobile app" OR "mHealth" OR "mobile health" OR "telehealth" OR "mobile technology" AND "Older adults" OR "Elderly" OR "Ageing population" OR "Older

Search string 1: population" OR "Aging" OR "Geriatric"

Search string 2: $\quad$ "Mobile application" OR "mobile app" OR "mHealth" OR "mobile health" OR "telehealth" OR "mobile technology" AND "Perspective*" OR "View" OR "Attitude" OR "Mindset" OR "Willingness" OR "Readiness" OR "Acceptability"

Search string 3:

"Mobile application"” OR "mobile app" OR "mHealth" OR "mobile health" OR "telehealth" OR "mobile technology" AND "Barrier" OR "Limitation*" OR "Difficulty" OR "Restriction*” OR "Drawback"

Search string 4:

"Mobile application"” OR "mobile app" OR "mHealth" OR "mobile health" OR "telehealth" OR "mobile technology" AND "Facilitate*" OR "Motivate*" OR "Promote*" OR "Help" OR "Ease" OR "Aid" 
PRISMA flow chart 1 will be used in the study selection process and will be updated once the review is completed (see online supplementary file 2 ).

\section{Eligibility criteria}

An article will be included when it:

- Describes or reports older adults' perspectives either their willingness or barriers or motivators towards the use of mobile applications in monitoring and managing their health condition.

- Is published in the English language.

- Contains only older population aged 60 and older as its study population.

- Is a peer-reviewed literature or grey literature.

- Is dated 1 January 2009-April 2019 (time frame of 10 years).

Studies that have been published from January 2009 to April 2019 were selected to be included in this study due to an immense growth reported in the number of mobile health applications download in the past 10 years with growth rate of more than $7 \%$ each year. ${ }^{40}$

An article will be excluded when it:

- Provides summaries and do not introduce any new knowledge (eg, literature review, scoping review, systematic review, topical review, commentaries, opinion papers).

\section{Stage 4: charting the data}

A data extraction framework will be developed to confirm study relevance and to extract study characteristics. Study characteristics to be extracted will include but not be limited to: standard bibliographical information (ie, authors, title, journal and year of publication), type and objectives of the review will be reported. For each article, we are going to extract the following data ${ }^{1}$ : characteristics of the study population, ${ }^{2}$ settings, ${ }^{3}$ characteristics of the mobile application used or tested and ${ }^{4}$ type of outcome assessed (ie, older adults' perspectives; their willingness, barriers and motivators towards the use of mobile applications to monitor and manage their health). A combination of EndNote V.X9 and Covidence software will be used to organise and track relevant data. We will use these softwares to ${ }^{1}$ remove duplicates ${ }^{2}$; document and

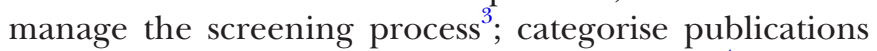
that meet the inclusion and exclusion criteria ${ }^{4}$; extract, organise and search related data and information from the publication content and $^{5}$ manage of full-texts version of included publications; including adding relevant notes that include key data extraction insights.

\section{Stage 5: collating, summarising and reporting the results}

Using the information collected from the data extraction form, the key characteristics of included studies will be summarised qualitatively and tabulated. All key findings will be described in narrative form. We will also be conducting a content analysis, identify emergent themes with regards to willingness, barriers and motivators from older adults. We will collect and identify objectives and gaps in our understanding of the current state or research. The discussion will be structured based on the themes that emerge.

\section{Stage 6: consultation with stakeholders}

This sixth stage of Arksey and O'Malley's framework ${ }^{34}$ is an optional component in conducting scoping reviews. We aim to engage with relevant stakeholders such as geriatricians, family medicine doctors, mobile applications developers, dietitians, psychologists and/or clinical psychologists to gain more insights into our data from different perspectives. A detailed design of consultation process will be created after stage five of the methodology (collating, summarising and reporting the results) has completed.

\section{Patient and public involvement}

As the review will use secondary data, patient and public will not be involved throughout the study.

Our study is meant to inform experts and stakeholders of the current state or issues concerning our topic. Following successful publishing of this protocol, we intend to submit a systematic scoping review to identify gaps within the research of older adults' perspectives towards the use of mobile application to monitor and manage health and identify what recommendations can be made to improve such gaps.

\section{Ethics and dissemination}

This scoping review protocol reports a comprehensive methodology. Since the data used are from publicly available sources, this study does not require ethical approval. Findings from this review will be disseminated through academic journals, seminars and conferences. We anticipate that our findings regarding older adults' perspectives towards the use of mobile applications to monitor and manage health conditions. This could guide the direction of future research and aid technology developers as well as health professionals working in the area of ageing and rehabilitation.

\section{Author affiliations}

${ }^{1}$ Center for Healthy Ageing and Wellness, Faculty of Health Sciences, Universiti Kebangsaan Malaysia, Kuala Lumpur, Wilayah Persekutuan Kuala Lumpur, Malaysia ${ }^{2}$ Faculty of Information Science and Technology, Universiti Kebangsaan Malaysia, Bangi, Selangor, Malaysia

${ }^{3}$ Faculty of Medicine, Universiti Kebangsaan Malaysia, Cheras, Kuala Lumpur, Wilayah Persekutuan Kuala Lumpur, Malaysia

Contributors NAA and AFML were responsible for developing the conception of the study. NAA wrote the manuscript with support from AFML and SS. AFML was responsible for reading and approving this manuscript's final version; giving final approval for the version that will be published, ensuring the integrity in all aspects of the work as well as making sure all research questions were addressed accordingly. SS was responsible for approving the design of the study; doing a thorough review to ensure intellectual content; reading and approving the final manuscript; giving the approval for the version that will be published and ensuring all research questions are analysed accordingly. SAMN and NMT contributed to the design of the study; acquired data about the research, read and approved the final manuscript and gave the final approval for the published version.

Funding This research received grant from the Ministry of Higher Education via the Dana Cabaran Perdana (DCP-2017-002/3). 
Competing interests None declared.

Patient and public involvement Patients and/or the public were not involved in the design, conduct, reporting or dissemination plans of this research.

Patient consent for publication Not required.

Provenance and peer review Not commissioned; externally peer reviewed.

Open access This is an open access article distributed in accordance with the Creative Commons Attribution Non Commercial (CC BY-NC 4.0) license, which permits others to distribute, remix, adapt, build upon this work non-commercially, and license their derivative works on different terms, provided the original work is properly cited, appropriate credit is given, any changes made indicated, and the use is non-commercial. See: http://creativecommons.org/licenses/by-nc/4.0/.

\section{ORCID iD}

Nurul Asilah Ahmad http://orcid.org/0000-0002-5727-1237

\section{REFERENCES}

1 Nations U. World population ageing 2019: highlights. New York: Department of Economic and Social Affairs PD, 2019.

2 Population and Demographics: Ageing [press release]. Department of statistics Malaysia, 2017.

3 Karim HA. The elderly in Malaysia: demographic trends. Med J Malaysia 1997;52:206-12.

4 Wilmoth JR. Demography of longevity: past, present, and future trends. Exp Gerontol 2000;35:1111-29.

5 Gordon B, Lindsay RMM, Riley J, et al. The contribution of public health and improved social conditions to increased life expectancy: an analysis of public awareness. $j$ community med health educ 2014

6 Davies AM. Epidemiology and the challenge of ageing. Int $J$ Epidemiol 1985;14:9-19.

7 Brayne $\mathrm{C}$. The elephant in the room - healthy brains in later life, epidemiology and public health. Nat Rev Neurosci 2007;8:233-9.

8 Brown G. The living end: the future of death, aging and immortality. London: Macmillan, 2008.

9 Americans. IoMUCotFHCWfO, inventorRetooling for an aging America: building the health care workforce. United States, 2008.

10 Acharya S, Ghimire S, Jeffers EM, et al. Health care utilization and health care expenditure of Nepali older adults. Front Public Health 2019;7:24.

11 Zhu X, Cai Q, Wang J, Xiaolong Z, Qiong C, Jin W, et al. Determinants of medical and health care expenditure growth for urban residents in China: a systematic review article. Iran J Public Health 2014;43:1597-604.

12 Wolff JL, Starfield B, Anderson G. Prevalence, expenditures, and complications of multiple chronic conditions in the elderly. Arch Intern Med 2002;162:2269-76.

13 Hoffman C, Rice D, Sung HY. Persons with chronic conditions. their prevalence and costs. JAMA 1996;276:1473-9.

14 Barnett K, Mercer SW, Norbury M, et al. Epidemiology of multimorbidity and implications for health care, research, and medical education: a cross-sectional study. Lancet 2012;380:37-43.

15 Hunsaker A, Hargittai E. A review of Internet use among older adults. New Media Soc 2018;20:3937-54.

16 OfN S. Internet users, UK: 2019. UK: Office for National Statistics, 2019.

17 Center PR. Smartphone ownership is growing rapidly around the world, but not always equally. United States: Pew Research Center, 2019.

18 Kontos E, Blake KD, Chou W-YS, et al. Predictors of eHealth usage: insights on the digital divide from the health information national trends survey 2012. J Med Internet Res 2014;16:e172.
19 Estacio EV, Whittle R, Protheroe J. The digital divide: examining socio-demographic factors associated with health literacy, access and use of Internet to seek health information. J Health Psychol 2019;24:1668-75.

20 Flynn KE, Smith MA, Freese J. When do older adults turn to the Internet for health information? findings from the Wisconsin longitudinal study. J Gen Intern Med 2006;21:1295-301.

21 Ryu S. Book review: mHealth: new horizons for health through mobile technologies: based on the findings of the second global survey on eHealth (global Observatory for eHealth series, volume 3). Healthc Inform Res 2012;18:231-3.

22 West JH, Hall PC, Hanson CL, et al. There's an APP for that: content analysis of paid health and fitness apps. J Med Internet Res 2012;14:e72.

23 Heart T, Kalderon E. Older adults: are they ready to adopt healthrelated ICT? Int J Med Inform 2013;82:e209-31.

24 Changizi M, Kaveh MH. Effectiveness of the mHealth technology in improvement of healthy behaviors in an elderly population-a systematic review. Mhealth 2017;3:51.

25 Syed ST, Gerber BS, Sharp LK. Traveling towards disease: transportation barriers to health care access. J Community Health 2013;38:976-93.

26 Agyemang-Duah W, Peprah C, Peprah P. Barriers to formal healthcare utilisation among poor older people under the livelihood empowerment against poverty programme in the Atwima Nwabiagya district of Ghana. BMC Public Health 2019;19:1185.

27 Fitzpatrick AL, Powe NR, Cooper LS, et al. Barriers to health care access among the elderly and who perceives them. Am J Public Health 2004;94:1788-94.

28 Doetsch J, Pilot E, Santana P, et al. Potential barriers in healthcare access of the elderly population influenced by the economic crisis and the troika agreement: a qualitative case study in Lisbon, Portugal. Int J Equity Health 2017;16:184.

29 Chang AY, Skirbekk VF, Tyrovolas S, et al. Measuring population ageing: an analysis of the global burden of disease study 2017. Lancet Public Health 2019;4:e159-67.

30 Anderson K, Burford O, Emmerton L. Mobile health Apps to facilitate self-care: a qualitative study of user experiences. PLOS One 2016;11:e0156164.

31 Free C, Phillips G, Galli L, et al. The effectiveness of mobile-health technology-based health behaviour change or disease management interventions for health care consumers: a systematic review. PLoS Med 2013;10:e1001362.

32 Muessig KE, Pike EC, Legrand S, et al. Mobile phone applications for the care and prevention of HIV and other sexually transmitted diseases: a review. J Med Internet Res 2013;15:e1.

33 Zhao J, Freeman B, Li M. Can mobile phone Apps influence people's health behavior change? an evidence review. J Med Internet Res 2016;18:e287.

34 Arksey H, O'Malley L. Scoping studies: towards a methodological framework. Int J Soc Res Methodol 2005;8:19-32.

35 Levac D, Colquhoun H, O'Brien KK. Scoping studies: advancing the methodology. Implement Sci 2010;5:69.

36 Daudt HML, van Mossel C, Scott SJ. Enhancing the scoping study methodology: a large, inter-professional team's experience with Arksey and O'Malley's framework. BMC Med Res Methodol 2013;13:48

37 Colquhoun HL, Levac D, O'Brien KK, et al. Scoping reviews: time for clarity in definition, methods, and reporting. J Clin Epidemiol 2014;67:1291-4.

38 Aromataris E, Munn Z. Joanna Briggs Institute Reviewer's Manual. The Joanna Briggs Institute, 2017.

39 Tricco AC, Lillie E, Zarin W, et al. PRISMA extension for scoping reviews (PRISMA-ScR): checklist and explanation. Ann Intern Med 2018;169:467-73.

40 Research2Guidance. mHealth APP economics 2017/2018: current status and future trends in mobile health, 2017. 